\title{
SENSOR LOCALIZATION USING ACOUSTIC DOPPLER SHIFT WITH A MOBILE ACCESS POINT
}

\author{
Richard J. Kozick \\ Bucknell University \\ Department of Electrical Engineering \\ Lewisburg, Pennsylvania 17837
}

Brian M. Sadler

\author{
Army Research Laboratory \\ 2800 Powder Mill Road \\ Adelphi, Maryland 20783
}

\begin{abstract}
The nodes in a sensor network are often deployed in random locations. However, most applications require that the location and/or orientation of the nodes be known, so post-deployment algorithms for self-localization of the sensor nodes are important. We consider using a mobile access point (AP) for sensor node localization in a randomly deployed sensor network. We focus on the particular case in which the sensors measure the acoustic Doppler shift in a tone that is emitted from the mobile AP. In addition to the acoustic emission, the mobile AP broadcasts a radio signal that contains AP position, velocity, timing, and parameters of the acoustic signal. We demonstrate that atmospheric turbulence has a significant impact on the accuracy of sensor localization, degrading performance by as much as two orders of magnitude relative to an ideal, planewave propagation model. We present Cramer-Rao bounds (CRBs) for sensor localization accuracy and compare the performance of algorithms to the CRBs under "cloudy" and "sunny" weather conditions.
\end{abstract}

Keywords: Sensor networks, sensor localization, location uncertainty, Cramér-Rao bound, atmospheric turbulence, atmospheric scattering.

\section{INTRODUCTION}

The nodes in a sensor network are often deployed in random locations, but most applications require that the location and/or orientation of the nodes be known. One approach for post-deployment localization of the sensor nodes uses beacons that are either external to the network (such as GPS) or deployed within the network. In this paper, we consider using a mobile access point (AP) for sensor node localization in a randomly deployed sensor network.

Deploying fixed beacons within the network enables a solution based on message passing between the nodes, e.g., see Moses et al. [1]. These beacons may be radio or another modality, such as acoustic, that takes advantage of the sen- sor capability. This approach assumes the communications network is pre-established, and requires sufficient density of beacons to be deployed, which raises the complexity of at least some of the nodes. Cevher and McClellan [2] proposed an approach to sensor localization that exploits an acoustic source that is external to the network and moves along a straight line path. The source trajectory is not known to the sensor nodes, so the source is not necessarily cooperating with the nodes. Each node tracks the angle of arrival (AOA) of the source and transmits the AOA tracks to a central information processor, then the central processor determines the node locations and orientations. This approach also assumes that the communications network is pre-established.

The use of a mobile AP for both communications and beaconing provides several advantages. The mobile AP can be used to localize many sensor nodes simultaneously in a broadcast mode, without requiring communications or synchronization between the nodes. The localization algorithms require only that the nodes receive the AP broadcast, and the AP can broadcast from several different beaconing positions as it moves. As a particular example, consider a network of acoustic sensors (microphones), where each node contains a microphone, a signal processor, and a narrowband radio. A helicopter that flies over the sensor network can serve as the mobile AP, and we assume that the AP has an accurate estimate of its own location and motion. The AP simultaneously broadcasts a radio message and an acoustic signal. The radio message contains timing, AP location and motion, and information about the acoustic signal. The sensor nodes receive this multi-modal transmission (radio and acoustic) and process it to self-localize. Acoustic experiments with a helicopter as the mobile AP are reported in [3].

At least three different quantities may be estimated at the sensor node for self-localization: Doppler shift, time delay (TDE), and angle of arrival (AOA). Although a single transmission modality would suffice, the multi-modal transmission has several advantages. If the acoustic broadcast is used alone, then the sensor nodes must communicate with each other since they do not know the AP timing, location / heading, and acoustic signal parameters. If the radio 
broadcast is used alone, then accurate TDE measurements require large time-bandwidth product waveforms. Receiving these would require a more sophisticated radio than the low-bandwidth, low-power radios that are typically used in sensor networks. Also, AOA measurements imply an antenna array, which again significantly raises the radio complexity.

In this paper, we consider self-localization of sensors based on measuring the acoustic Doppler shift in a tone that is emitted from a mobile access point. We assume that the sensor node knows the location and heading of the AP as well as the frequency of the acoustic tone (these are contained in the radio signal that is transmitted from the AP). We consider Doppler shift alone for the sensor localization because the processing is very simple (spectral line estimation) and does not require a microphone array. Combining the Doppler estimate with TDA and/or AOA will improve localization accuracy, but these considerations will be reported elsewhere. We will focus in this paper on the significant impact that atmospheric turbulence has on the accuracy of Doppler estimation and, consequently, on the accuracy of sensor localization. We present Cramér-Rao bounds (CRBs) on localization accuracy as a function of the atmospheric conditions, and we compare the performance of algorithms to the localization bounds. A significant conclusion is that compared with ideal propagation conditions (perfect plane waves), the turbulence degrades the localization accuracy by as much as two orders of magnitude.

\section{MODEL AND SIGNAL PROCESSING}

\subsection{Turbulence model and Doppler estimation}

We use a statistical model for turbulent scattering of sound in the atmosphere developed by Wilson, Collier, and others [5]-[6]. The model is summarized in [7]-[8], and the effect of turbulence on an acoustic tone is to scatter a portion of the energy from the pure tone emitted by the source into a zero-mean random process, as illustrated in the power spectral density (PSD) in Figure 1. The saturation parameter, denoted by $\Omega \in[0,1]$, defines the fraction of average signal power that is scattered from the mean into the random component. In Figure 1, the frequency $f_{d}$ is the Doppler shift, $2 \mathcal{N}_{o}$ is the PSD of the AWGN (complex, circular, Gaussian random process with zero mean), $S$ is the average energy of the received signal, $B$ is the processing bandwidth, and $B_{v}$ is the bandwidth of the scattered component. This implies that the autocorrelation function of the scattered component has coherence time on the order of $1 / B_{v}$ sec. Sampling the sensor signal at the rate $F_{s}=B$ samples per second for $T$ seconds yields $M=\lfloor B T\rfloor$ samples, which we denote as $\widetilde{\mathbf{z}}=\left[\begin{array}{lll}\widetilde{z}(0) & \ldots & \widetilde{z}\left((M-1) T_{s}\right)\end{array}\right]^{T}$. This vector has a complex Gaussian distribution with mean and covariance

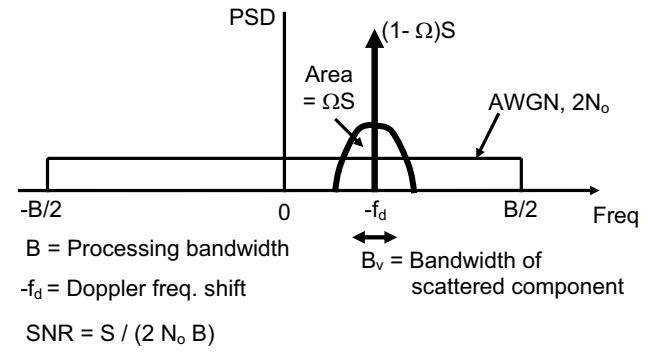

Fig. 1. PSD $G_{\tilde{z}}(f)$ for the sensor signal with Doppler shift and turbulent scattering.

matrix

$$
\begin{aligned}
\widetilde{\mathbf{z}} \sim \mathbf{C N}\left(e^{j \theta} \sqrt{(1-\Omega) S} \mathbf{a},\right. \\
\left.(\Omega S) \mathbf{R}_{\tilde{v}} \circ\left(\mathbf{a a}^{H}\right)+\left(2 \mathcal{N}_{o} B\right) \mathbf{I}\right),
\end{aligned}
$$

where

$\mathbf{a}^{T}=\left[1, \exp \left(-j 2 \pi f_{d} / B\right), \ldots, \exp \left(-j 2 \pi(M-1) f_{d} / B\right)\right]$,

$\mathbf{R}_{\tilde{v}}$ is the covariance matrix of the samples of the scattered process, o denotes element-wise product, $(\cdot)^{H}$ denotes Hermitian transpose, and $\mathbf{I}$ is the identity matrix.

The scattering causes fluctuations in the signal energy at the sensor. Ideal propagation corresponds to $\Omega=0$, in which case the emitted signal arrives as a deterministic tone in AWGN. The Gaussian model for the scattering is somewhat idealized, but it has been shown experimentally to be accurate in many cases of interest [9].

The "saturation" parameter, $\Omega$, varies with the atmospheric conditions (sunny/cloudy), source frequency $\left(f_{s}\right.$ in $\mathrm{Hz}$ ), and the source range ( $r$ in meters) as [7]

$$
\Omega=1-\exp \left(-\kappa f_{s}^{2} r\right)
$$

where $\kappa \approx 8.1 \times 10^{-7}$ for mostly sunny conditions and $\kappa \approx 2.8 \times 10^{-7}$ for mostly cloudy conditions. Note from (2) that the scattering becomes more severe at higher source frequencies and larger ranges. Typical aeroacoustic frequencies are in the range from 30 to $200 \mathrm{~Hz}$ and ranges are from 5 to $500 \mathrm{~m}$, and for these values $\Omega$ spans the entire range from 0 to 1 .

In [8], we have studied CRBs and algorithms for Doppler estimation in turbulence. Two algorithms for Doppler estimation are summarized here because they are used in this paper to perform sensor self-localization. The periodogram (P-GRAM) is the maximum likelihood estimator with no scattering $(\Omega=0)$, and is given by

$\widehat{f}_{d}=\underset{f_{d}}{\operatorname{argmax}}\left|\sum_{n=0}^{M-1} \widetilde{z}\left(n T_{s}\right) \exp \left(j 2 \pi f_{d} n T s\right)\right|^{2}$. For

the case of full scattering $(\Omega=1)$, the signal component in (1) is a random process with unknown covariance matrix 
$\mathbf{R}_{\tilde{v}}$. For the case in which $\mathbf{R}_{\tilde{v}}$ is real-valued, Toeplitz, and unknown, Besson and Stoica [13] proposed the following estimator:

$\widehat{f}_{d}=\underset{f_{d}}{\operatorname{argmax}} \operatorname{Re}\left\{\sum_{m=1}^{M-1} \widehat{r}_{\tilde{z}}[m]^{2} \exp \left(j 4 \pi f_{d} m T s\right)\right\}$,

where $\widehat{r}_{\tilde{z}}[m]$ is a consistent estimate of the $m^{\text {th }}$ lag of the sensor signal autocorrelation. We label this estimator

$\mathrm{C}^{2}$-GRAM because it is similar to the correlogram.

\subsection{Sensor localization with Doppler}

We assume that the mobile AP emits an acoustic tone with frequency $f_{s}$ at times $t_{1}, \ldots, t_{N}$. The location $\mathbf{x}_{n}=\left(x_{n}, y_{n}\right)$ and velocity $\dot{\mathbf{x}}_{n}=\left(\dot{x}_{n}, \dot{y}_{n}\right)$ of the AP source are known to the sensors at each time $t_{n}$, and the frequency $f_{s}$ is also known via the radio channel. We consider estimating the unknown sensor location, $\mathbf{x}_{o}=\left(x_{o}, y_{o}\right)$, based on processing the estimated Doppler shifts of the acoustic tone for the $N$ emissions from the mobile AP.

The range vector from source to sensor is $\mathbf{r}_{n}=\mathbf{x}_{o}-\mathbf{x}_{n}$, the range is $r_{n}=\left\|\mathbf{r}_{n}\right\|$, and the source speed is $V_{n}=\left\|\dot{\mathbf{x}}_{n}\right\|$, where $\|\cdot\|$ denotes the Euclidean norm of the vector. The angles $\alpha_{n}$ between the source velocity vector and the range vector and the source azimuth $\phi_{n}$ are

$$
\begin{aligned}
\cos \alpha_{n} & =\frac{\mathbf{r}_{n} \cdot \dot{\mathbf{x}}_{n}}{r_{n} V_{n}} \\
\cos \phi_{n} & =\frac{x_{n}-x_{o}}{r_{n}}, \quad \sin \phi_{n}=\frac{y_{n}-y_{o}}{r_{n}},
\end{aligned}
$$

where $\cdot$ denotes the inner product between vectors. The Doppler-shifted frequency observed at the sensor node corresponding to the $\mathrm{AP}$ transmission at time $t_{n}$ is [10]

$$
\begin{aligned}
f_{n} & =f_{s}\left(1+\frac{V_{n} \cos \alpha_{n}}{c}\right) \\
& =f_{s}+\left(\frac{f_{s}}{c}\right) \frac{\left(x_{o}-x_{n}\right) \dot{x}_{n}+\left(y_{o}-y_{n}\right) \dot{y}_{n}}{\left[\left(x_{o}-x_{n}\right)^{2}+\left(y_{o}-y_{n}\right)^{2}\right]^{1 / 2}} \\
& =f_{s}+\left(\frac{f_{s}}{c}\right) g\left(\mathbf{x}_{o} ; \mathbf{x}_{n}, \dot{\mathbf{x}}_{n}\right) .
\end{aligned}
$$

In (5)-(7), the unknowns are the sensor node location $\mathbf{x}_{o}=$ $\left(x_{o}, y_{o}\right)$, while the quantities $f_{s}, x_{n}, y_{n}, \dot{x}_{n}, \dot{y}_{n}$ are known because they are transmitted via radio from the AP to the sensor node. We assume the acoustic velocity $c$ is known. This can be obtained from measurements of meteorological parameters such as temperature, humidity, and so on. The function $g\left(\mathbf{x}_{o} ; \mathbf{x}_{n}, \dot{\mathbf{x}}_{n}\right)$ is defined in (7) to emphasize that the sensor location $\mathbf{x}_{o}$ is unknown while the source parameters $\mathbf{x}_{n}, \dot{\mathbf{x}}_{n}$ are known.

Given $N$ estimates of the measured frequency at the sensor, we can estimate the Doppler shifts, $\Delta f_{n}=f_{n}-f_{s}$, and then use weighted, nonlinear least-squares to estimate the sensor location, $\mathbf{x}_{o}$ :

$$
\widehat{\mathbf{x}}_{o}=\underset{\mathbf{x}_{o}}{\arg \min } \sum_{n=1}^{N} \frac{1}{\sigma_{n}^{2}}\left[\Delta f_{n}-\frac{f_{s}}{c} g\left(\mathbf{x}_{o} ; \mathbf{x}_{n}, \dot{\mathbf{x}}_{n}\right)\right]^{2}
$$

Next, we combine the statistical model for the time samples at the sensor in (1) with the dependence of the Doppler shift on the sensor location in (5)-(7). That is, for each $t_{n}$, we have a vector of samples, $\widetilde{\mathbf{z}}_{n}$, modeled by (1), with average signal power $S_{n}$, saturation $\Omega_{n}$, and Doppler shift $f_{n}$ given by (5)-(7). We model $\widetilde{\mathbf{z}}_{1}, \ldots, \widetilde{\mathbf{z}}_{N}$ as statistically independent, which is reasonable if the AP locations are separated in space so that the scattering is independent.

We have derived the CRB on the sensor location, $\mathbf{x}_{o}$, using standard results, e.g., see Kay [11]. In addition, we have simulated the performance of sensor localization algorithms as follows.

1. Generate samples $\widetilde{\mathbf{z}}_{1}, \ldots, \widetilde{\mathbf{z}}_{N}$ using the model (1).

2. Estimate the Doppler shifts $\widehat{f}_{1}, \ldots, \widehat{f}_{N}$ using the PGRAM or $\mathrm{C}^{2}$-GRAM algorithms described above.

3. Estimate the sensor location, $\widehat{\mathbf{x}}_{o}$, using the nonlinear least-squares method in (8).

Algorithm performance is compared with the CRB in the following section. We will also show the CRBs and algorithm performance for the case of no scattering $(\Omega=0)$, illustrating the large impact that turbulent scattering has on the achievable performance.

\section{EXAMPLES}

We present an example of sensor self-localization in this section. The framework we have presented in this paper is useful to design trajectories for the mobile AP so that the sensors achieve a desired level of accuracy in self-localization. Since we include the effects of turbulent scattering by the atmosphere, our results are much more realistic than an ideal plane wave propagation model.

The sensor is located at the origin $\mathbf{x}_{o}=(0,0)$, the speed of sound is $c=335 \mathrm{~m} / \mathrm{s}$, and the mobile AP source speed is $V_{n}=0.2 c$ (for all $n$ ). The source travels along a circular path around the sensor with radius $300 \mathrm{~m}$, emitting a tone with $f_{s}=100 \mathrm{~Hz}$ at $N=3$ locations, with azimuth $\phi_{1}=-90^{\circ}, \phi_{2}=0^{\circ}$, and $\phi_{3}=90^{\circ}$. The source trajectory is tangential to the sensor, so $\alpha_{n}=90^{\circ}$ for $n=1,2,3$. In Figure 1, the processing bandwidth is $B=40 \mathrm{~Hz}$, the scattering bandwidth $B_{v}=1 \mathrm{~Hz}$, the SNR is $21 \mathrm{~dB}$, and the observation time is $T=2 \mathrm{sec}$ with $M=80$ samples. We consider two weather conditions for the range $300 \mathrm{~m}$ and frequency $100 \mathrm{~Hz}$, and (2) yields saturation values $\Omega=0.57$ 
for mostly cloudy and $\Omega=0.91$ for mostly sunny conditions.

Figure 2 shows the CRB ellipse (defined by $\mathbf{x}^{T} \mathbf{J} \mathbf{x}=1$ where $\mathbf{J}$ is the Fisher information matrix for the sensor location) and a scatter plot of the sensor location estimates for 200 runs. The $C^{2}$-GRAM algorithm is used for Doppler estimation. Comparing parts (a) and (b) of Figure 2, sunny conditions reduce the accuracy relative to cloudy conditions due to increased turbulent scattering. The root-mean-squared error (RMSE) performance of the algorithm is about four times $\sqrt{\mathrm{CRB}}$, with accuracy on the order of several meters. Figure 3 shows corresponding results using the P-GRAM algorithm for Doppler estimation. Note that P-GRAM is less accurate than $\mathrm{C}^{2}$-GRAM, which is expected since PGRAM is optimum when $\Omega=0$.

We repeated the simulation with no scattering, $\Omega=$ 0 . For this case, the $\mathrm{CRB}$ on sensor location is $\sqrt{\mathrm{CRB}}=$ $0.03 \mathrm{~m}$, and the RMSE performances of the algorithms are equal to the $\sqrt{\mathrm{CRB}}$. Thus turbulent scattering degrades the achievable sensor localization accuracy by a factor of about 100 relative to ideal propagation.

\section{REFERENCES}

[1] R. L. Moses, D. Krishnamurthy, R. Patterson, "A selflocalization method for wireless sensor networks," Eurasip Jrnl. Appl. Sig. Proc., vol. 4, pp. 348-358, 2003.

[2] V. Cevher and J.H. McClellan, "Sensor array calibration via tracking with the extended Kalman filter," Proc. ICASSP 2001, Salt Lake City, UT, May 7-11, 2001.

[3] T. R. Damarla, V. Mirelli, "Sensor localization using helicopter acoustic and GPS data," Proc. of SPIE, vol. 5417, pp. 336-340, April 2004.

[4] B.M. Sadler, R.J. Kozick, and L. Tong, "Multi-modal sensor localization using a mobile access point," Proc. ICASSP 2005, Philadelphia, March 2005.

[5] S.L Collier and D.K. Wilson, "Performance bounds for passive arrays operating in a turbulent medium: Plane-wave analysis," J. Acoust. Soc. Am., Vol. 113, No. 5, pp. 2704-2718, May 2003.

[6] D. E. Norris, D. K. Wilson, and D. W. Thomson, “Atmospheric scattering for varying degrees of saturation and turbulent intermittency," J. Acoust. Soc. Am., vol. 109, pp. 1871-1880, 2001.

[7] R.J. Kozick, B.M. Sadler, and D.K. Wilson, "Signal Processing and Propagation for Aeroacoustic Sensor Networking," Ch. 13 in Distributed Sensor Net- works, S.S. Iyengar and R.R. Brooks (Eds.), Chapman \& Hall/CRC Press, Boca Raton, Florida, 2005.

[8] R. J. Kozick, B. M. Sadler, "Performance of Doppler estimation for acoustic sources with atmospheric scattering," Proc. ICASSP'04, pp. 381-384, May 2004.

[9] G. A. Daigle, J. E. Piercy, and T. F. W. Embleton, "Line-of-sight propagation through atmospheric turbulence near the ground," J. Acoust. Soc. Am., vol. 74, pp. 1505-1513, 1983.

[10] Y. T. Chan, J. J. Towers, "Passive localization from Doppler-shifted frequency measurements," IEEE Trans. Sig. Proc., vol. 40, no. 10, pp. 2594-2598, Oct. 1992.

[11] S.M. Kay, Fundamentals of Statistical Signal Processing: Estimation Theory, Prentice-Hall, 1993.

[12] P.M. Schultheiss and E. Weinstein, "Estimation of differential Doppler shifts," J. Acoust. Soc. Am., vol. 66, no. 5, pp. 1412-1419, Nov. 1979.

[13] O. Besson and P. Stoica, "On frequency offset estimation for flat-fading channels," IEEE Communications Letters, vol. 5, no. 10, pp. 402-404, Oct. 2001. 


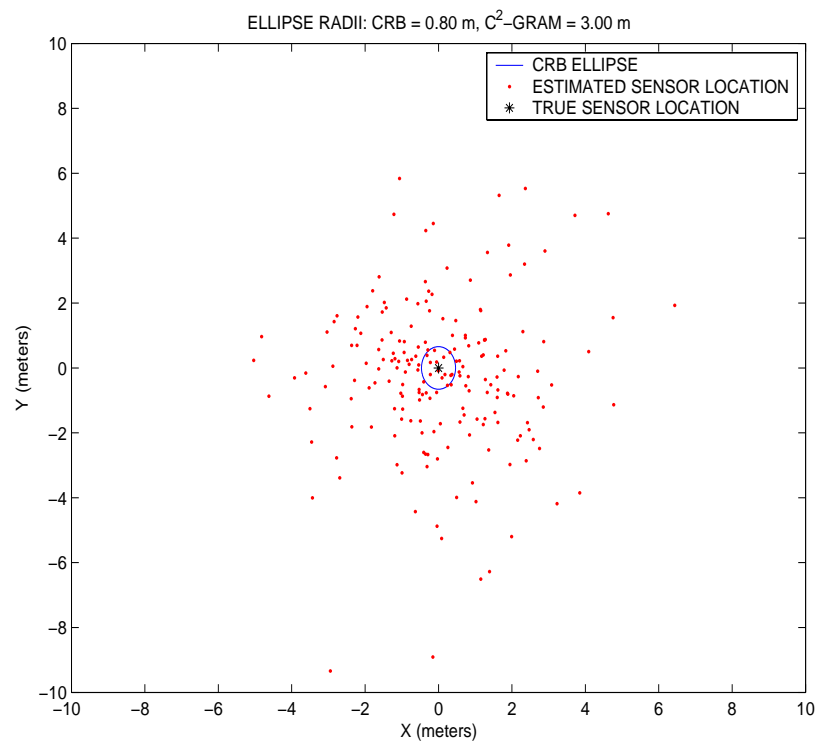

(a)

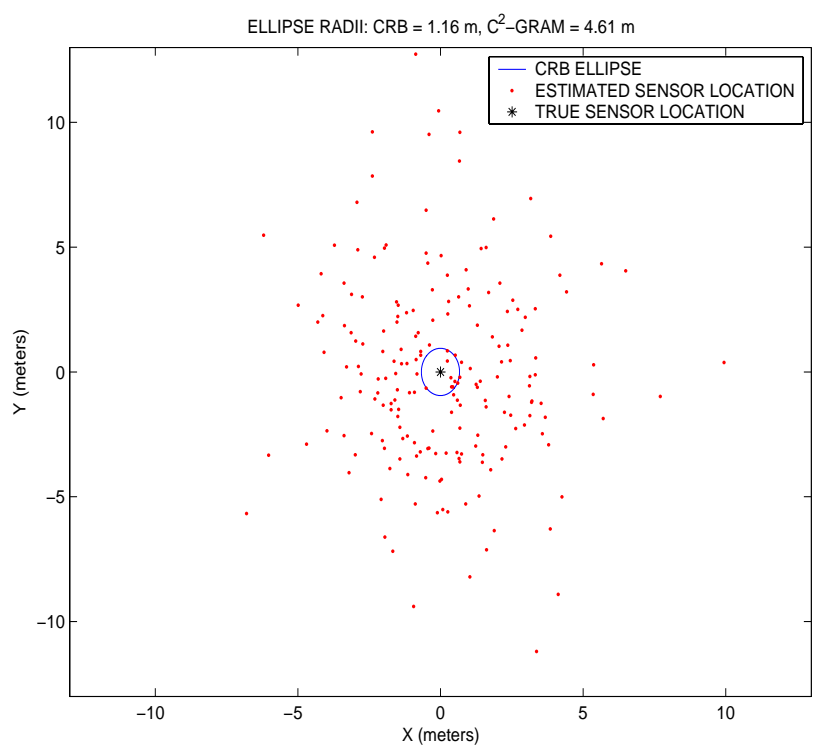

(b)

Fig. 2. CRB ellipse for sensor location and scatter plot of estimated sensor location for 200 runs, with $C^{2}$-GRAM Doppler estimator and circular source trajectory around sensor with $N=3$ transmissions. (a) Mosty cloudy and (b) mostly sunny conditions.

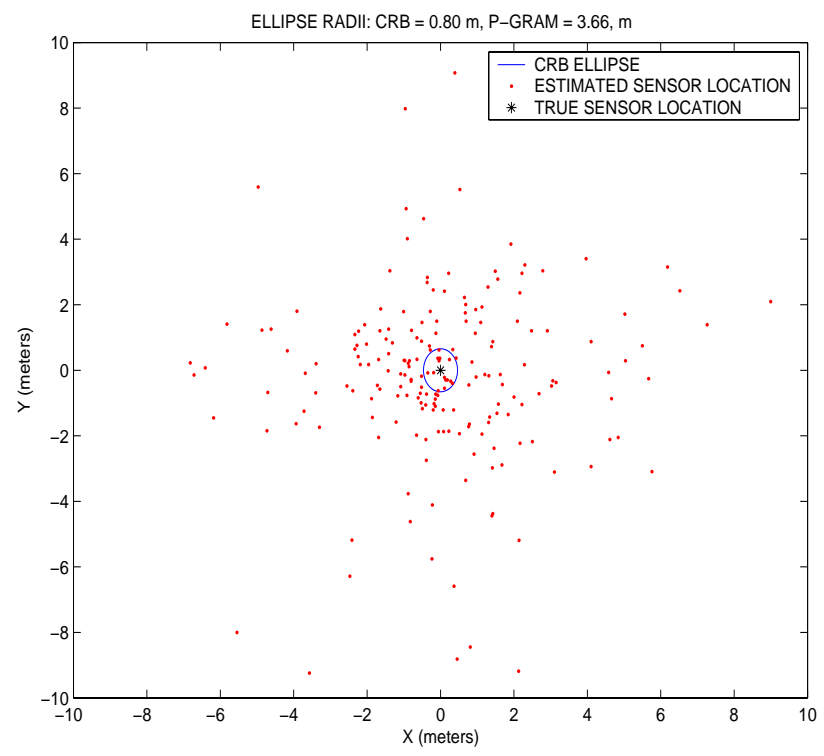

(a)

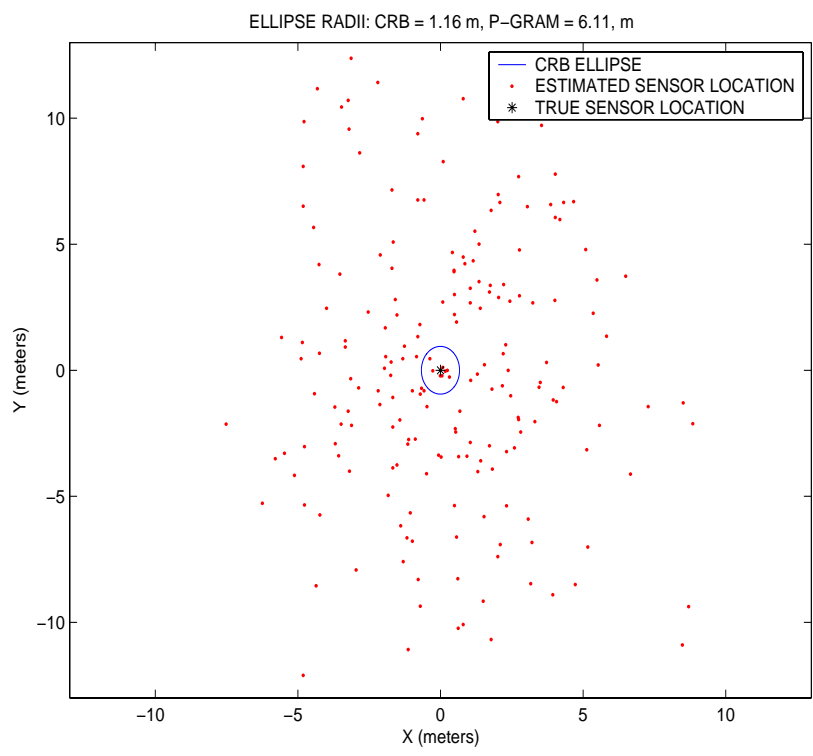

(b)

Fig. 3. CRB ellipse for sensor location and scatter plot of estimated sensor location for 200 runs, with P-GRAM Doppler estimator and circular source trajectory around sensor with $N=3$ transmissions. (a) Mosty cloudy and (b) mostly sunny conditions. 